sen sein kann, und zu zeigen, wie es gelingt, dieses zur Mehrung unseres kollektiven Wissenspools nicht nur überzeugend, sondern auch überprüfbar zusammenzutragen. Und letztlich ist diese Sammlung - einschließlich des Gedichtes auf der allerletzten Seite - auch ein kleines gelungenes Kunstwerk.

Für Menschen mit Interesse an spirituellen Fragen oder die - für sich oder ihre Klientlnnen - nach einer mystischen Praxis suchen, die in einem modernen Kontext praktiziert werden kann, also vor dem Hintergrund unter- schiedlichster spiritueller Orientierungen und im heutigen Wissen um psychologische, gruppenrelevante und therapeutische Aspekte des menschlichen Seins, bietet das Buch einen reichen Fundus an vielfältigen Erfahrungsberichten und tiefen Einsichten, um sich ein Bild von den vielschichtigen Möglichkeiten des Übungsweges Authentic Movement zu machen.

\section{Marion Reuter}

DOI 10.2378/ktb2018.art25d

\title{
Helga Krüger-Kirn / Bettina Schroeter (Hrsg.): Verkörperungen von Weiblichkeit. Gendersensible Betrachtungen körperpsychotherapeutischer Prozesse
}

\author{
Psychosozial-Verlag, 2017, Gießen, 324 Seiten, 34,90€ (D)
}

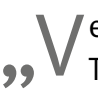
erkörperungen von Weiblichkeit“"ist ein Titel, der all diejenigen, die sich mit Körper und Psychotherapie befassen, neugierig macht. Sind die Lebenserfahrungen von Frauen anders im Körper verankert als die von Männern? Die 2013 in Marburg stattgefundene Tagung „Das andere Wissen“ der Deutschen Gesellschaft für Körperpsychotherapie (DGK) versuchte, dieser Frage nachzugehen, und befasste sich mit den spezifisch weiblichen Bedingungen von Körpererfahrung, Körperwahrnehmung und Körperausdruck. Die Herausgeberinnen des Tagungsbandes, Bettina Schroeter und Helga Krüger-Kirn, betonen in ihrer Einleitung die gesellschaftliche Relevanz der Thematik, die über die individuelle Perspektive in den soziokulturellen Raum hinein wirkt.

Der Band enthält alle Tagungsbeiträge. Das Inhaltsverzeichnis ist in drei Abschnitte unterteilt, zu denen jeweils mehrere Beiträge gehören. Auf diese Weise haben die LeserInnen dieser Publikation schon durch das Inhaltsverzeichnis einen Kompass, der durch die Ta- gungsthemen führt. Die Themen sind unter folgenden Großtiteln zusammengefasst: I Frauenkörper - Sprache - Körperwissen, II Schönheit Sexualität - Mutterschaft, III Phänomene moderner Weiblichkeit im körperpsychotherapeutischen Prozess.

Der erste Abschnitt enthält eher konzeptionelle Beiträge. Bettina Schroeter geht es darum, dass Frauen den Zugang zu ihrem Körper finden, um sich in ihrem „eigenen Körper zu beheimaten“ (S.36). Helga Krüger-Kirn zeigt in ihrem Beitrag, dass das „emanzipatorische Potential der Körperpsychotherapie (...) in der Sichtbar- und Sprechbarmachung weiblicher Körperlichkeit“ liegt (S. 59).

Susanne Maurer warnt davor, Körperwahrnehmungen in einer undifferenzierten Weise zu deuten oder zu verstehen. Der Artikel von Sabine Schrem beschreibt ein Projekt, in dem Körperpsychotherapeutinnen sich auf die 
Suche nach ihren beruflichen „Ahninnen“ machen und so an therapeutischer Verankerung und Selbstbewusstsein gewinnen.

Im zweiten Teil beschreibt Angela von Arnim gut belegt den Trend zu plastischer Chirurgie bei Frauen, den sie besorgniserregend findet, da oft hinter dem Wunsch nach einer solchen Operation ein psychischer Konflikt steht, der auf diese Weise natürlich nicht gelöst wird, sondern möglicherweise noch verschärft. Thea Rytz beschreibt die Auswirkung von sexistischer Werbung auf das Körpergefühl und die verzerrte Körperwahrnehmung bei Frauen und leitet nachvollziehbar ab, wie dadurch Essstörungen entstehen können. Anke Abraham stellt die weibliche Sexualität in einen soziologischen Rahmen, der sowohl die Überformung der Sexualität durch soziokulturelle Normen beschreibt als auch die Notwendigkeit der Berücksichtigung von leiblichen Dispositionen hervorhebt. Paula Diederichs, Eva Bogensprenger-Herzel und Anja Weiffen zeigen uns, dass Schwangerschaft und Mutter-Werden für jede Frau eine „Superherausforderung“ darstellen, in der die Frau verständnisvoll umsorgt werden muss und ihren eigenen Weg finden darf. Sie zeigen aber auch, dass die soziokulturellen Bedingungen heute dafür nicht günstig sind.

Im dritten Teil beschreibt Doris Lange in ihrem Beitrag die Therapie von Frauen mit Burn-out-Symptomatik, nachdem sie zuvor auf die besonderen neurobiologischen Verarbeitungsmodi von Frauen hingewiesen hat. Mit der Funktionellen Entspannung verhilft sie den Patientinnen zu einer vertieften leiblichen Eigenwahrnehmung, die diese Frauen dazu befähigt, für sich Lösungsstrategien zu entwickeln. Cornelia Richter-Grimm und Anna WillachHolzapfel setzen sich mit den Spezifika weiblicher Aggression auseinander. Sie wünschen sich statt einer verdeckten eine offene Aggressivität, die auch die Verantwortung dafür übernimmt. Angelika Weirauch zeigt auf, dass
Frauen mit Behinderungen öfter Opfer von Gewalterfahrungen werden als Frauen ohne Behinderung. Sie fordert einen sensiblen Umgang mit Behinderung. Christina Angelini weist in ihrem Beitrag auf die besondere Situation von Frauen aus Nahost hin, die geschlechtsspezifische Gewalt erfahren haben, und beschreibt ihre Arbeit mit körperpsychotherapeutischen und anderen Methoden zur Traumaverarbeitung. Dorothea Haffner stellt ihre Form der Tanztherapie vor und beschreibt ein Tanzprojekt, das für traumatisierte Frauen hilfreich war.

Die Beiträge in diesem Band sind sehr unterschiedlich und tragen damit dem großen Spektrum der therapeutischen Arbeit mit Frauen Rechnung, aber auch der Fülle an interessanten Frauen-Persönlichkeiten, die sich in der Arbeit für dieses Buch zusammengefunden haben. So reicht der Bogen von eher konzeptionellen Reflektionen über die Darstellung spezifisch weiblichen Umgangs mit dem Körper bis hin zu einfühlsam beschriebenen therapeutischen Ansätzen. Es macht Freude, dieses Buch immer wieder zur Hand zu nehmen und den Frauen bei ihrer Arbeit über die Schulter zu schauen.

So unterschiedlich die Beiträge sein mögen, so sind sie doch von einem gemeinsamen Tenor durchzogen: Es geht den Autorinnen um einen respektvollen Umgang mit spezifisch weiblichen Körpererfahrungen, die in der (therapeutischen) Arbeit mit Frauen kenntnisreich berücksichtigt werden müssen. Insofern ist der Band nicht nur für Frauen spannend, für die eine Selbstvergewisserung bezüglich ihrer leiblichen Erfahrung stattfinden kann, sondern besonders auch für Männer, die Verkörperungen von Weiblichkeit differenzierter wahrnehmen und besser verstehen wollen.

Dipl. Psych. Gabriele Martin

DOI 10.2378/ktb2018.art26d 